\title{
1 The Culturome of the Human Nose Habitats Reveals Individual Bacterial Fingerprint Patterns
}

2

3

4 Ursula Kaspar ${ }^{1}$, André Kriegeskorte ${ }^{1}$, Tanja Schubert ${ }^{2}$, Georg Peters ${ }^{1}$, Claudia Rudack ${ }^{2}$, Dietmar H.

5 Pieper $^{3}$, Melissa Wos-Oxley ${ }^{3}$, Karsten Becker ${ }^{1 *}$

6

$7 \quad 1 \quad$ Institute of Medical Microbiology, University Hospital Münster, Münster, Germany

82 Department of Otorhinolaryngology Head and Neck Surgery, University Hospital

9 Münster, Münster, Germany

103 Research Group Microbial Interactions and Processes, Helmholtz Centre for Infection Research, 11 Braunschweig, Germany

12

13 Running Head: Human Nose Culturome

14

$15 *$ Corresponding author: Karsten Becker, Institute of Medical Microbiology, University Hospital 16 Münster, Domagkstr. 10, 48149 Münster, Germany, Telephone: +49 251 8355375, Fax: +492518355350, Email: kbecker@uni-muenster.de

18 


\section{Summary}

The complex anatomy of the human nose might offer distinct microbial niches. Microbiota composition may affect nose inflammatory diseases and Staphylococcus aureus carriage. Considering different nasal cavity locations, microbial colonisation was analysed across individuals exhibiting chronic nasal inflammatory diseases $(n=18)$ and those without local inflammation signs $(n=16)$. Samples were collected systematically during surgery and examined by an extensive culture-based approach and, for a subset, by 16S rRNA gene community profiling. Cultivation yielded 141 taxa with members of Staphylococcus, Corynebacterium and Propionibacterium as most common isolates comprising the nasal core culturome together with Finegoldia magna. S.aureus was most frequently found in association with Staphylococcus epidermidis and Propionibacterium acnes and the posterior vestibules were redefined as S.aureus' principle habitat. Culturome analysis revealed host-specific bacterial "fingerprints" irrespective of host-driven factors or intranasal sites. Comparisons between cultivable and molecular fingerprints demonstrated that only a small fraction of phylotypes (6.2\%) was correlated. While the total number of different phylotypes was higher in the molecular data set, the total number of identifications down to the species level was higher in the culturomic approach. To determine host-specific microbiomes, the advantages of molecular approaches should be combined with the resolution and reliability of species identification by culturomic analyses. 
Mammalian body surfaces provide diverse habitats for a large number of microbial species and numerous studies have described the diversity and complexity of human microbial ecosystems (Grice and Segre, 2012; The Human Microbiome Project Consortium, 2012) and their impact for health and disease (Kosiewicz et al., 2011; Pflughoeft and Versalovic, 2012). In contrast to the orogastrointestinal tract, the surfaces of the human nasal cavity represent a less studied ecosystem. First cultureindependent analyses revealed a densely populated habitat for many bacterial species (Frank et al., 2010; Wos-Oxley et al., 2010; Camarinha-Silva et al., 2012) where age was shown to significantly shape the composition of the microbiota (Camarinha-Silva et al., 2014).

Besides questions concerning etiopathogenic links between the nasal microbiota and chronic nasal disease entities such as rhinosinusitis and polyposis, the human nose as principle habitat of the major opportunistic pathogen Staphylococcus aureus warrants studies on the composition of nasal microbiota in healthy and diseased individuals. S. aureus permanently colonises approximately 2030\% of the human population (van Belkum et al., 2009; Sollid et al., 2014). Though the species mostly acts as an asymptomatic commensal in the nose, its colonisation can be source and risk factor for subsequent infections (Kluytmans et al., 1997; von Eiff et al., 2001; Wertheim et al., 2004). The global burden by healthcare-, community- and livestock-associated methicillin-resistant S. aureus (MRSA) clonal lineages aggravates its clinical and socioeconomic impact by drastically limiting our therapeutic armamentarium (Köck et al., 2010, 2013; Mediavilla et al., 2012; Dantes et al., 2013). Colonisation of S. aureus in the nasal cavity happens under the influence of numerous co-occurring species (Frank et al., 2010; Wos-Oxley et al., 2010) that have been assumed to may have beneficial, mutual or antagonistic effects on S. aureus regarding its establishment and permanent residence in the nose (Uehara et al., 2000; Lina et al., 2003; Frank et al., 2010; Yan et al., 2013). Anyway, most microbiome studies are exclusively based on molecular data (Frank et al., 2010; Lemon et al., 2010; Yan et al., 2013), without validating results by comparison to a different method. human body and its outer ecological environment. Filtering, warming and humidifying the inhaled air, 
it functions as a passage to the lungs and the paranasal sinuses and exhibits a high diversity of anatomical sites on a narrow space (Geurkink, 1983; Wagenmann and Naclerio, 1992). Skin-like squamous epithelium containing vibrissae and sebaceous or sweat glands can be found in the anterior vestibules whereas the deeper nasal cavity is characterised by pseudostratified columnar ciliated epithelium (respiratory epithelium) being covered with a mucus blanket that is in constant movement (Geurkink, 1983; Cheesman and Burdett, 2011). In this posterior part of the nasal cavity, three nasal meatuses (superior, middle and inferior meatus) are found located beneath each of the corresponding nasal conchae. The knowledge about these entirely different anatomical structures appearing close to each other inevitably leads to the hypothesis of defined ecological niches and microbial structures. However, this factor has been widely disregarded hitherto (Glück, 2001) and studies of the human nasal microbiome either focused on one anatomical site (Feazel et al., 2012) or neglected mentioning the exact site of sampling (Gittelman et al., 1991; Frank et al., 2010; Alvarez et al., 2013). Moreover, especially in literature concerning $S$. aureus colonisation, the anterior nares are often equated to the complete nasal vestibule. In fact, the nasal vestibule (vestibulum nasi) comprises the area between the nares (atrium) and the limen nasi as gradient zone to the respiratory epithelium behind the vestibules. Here, we present the first study based on an in depth cultivation approach to catalogue the nasal microbiota that considers the different anatomical sites of the nasal cavity as possible distinct ecological niches with different microbial communities. We further elucidated the influence of chronic inflammatory conditions of nasal epithelial surfaces on the bacterial composition. Members of the culturome were identified by molecular and mass spectrometric techniques down to the species level. sequencing. 
Results

Study group and collection of specimens

A total of 34 ENT patients (22 males and 12 females; 19 to 78 years) were enrolled comprising a first group with 16 individuals without any signs of nasal and systemic inflammation and a second group with 18 patients suffering from chronic inflammatory diseases. Of the second group, 10 patients were diagnosed with chronic sinusitis (CS), 1 showed a chronic polyposis (CP) and 7 suffered from both. None of the patients had been taking antibiotics for at least 6 weeks prior to surgery, but 10 of them had frequently been using nose sprays that also contained cortisone in 7 of the cases. Further information on patient metadata is given in Supporting Information Table S1.

Bacterial diversity of the culturome

101 By cultivation, a total of 141 different bacterial taxa were identified including all patients and nasal 102 habitats tested (Table S2). Of these, 139 (98.6\%) could be differentiated down to the species level and two to the genus level. Per patient, 15.2 different taxa were identified on average (Table 1). Most prevalent species were Staphylococcus epidermidis and the facultative anaerobe Propionibacterium acnes, both found in $97.1 \%$ of patients followed by Corynebacterium accolens and Corynebacterium tuberculostearicum each colonising $64.7 \%$ of the individuals. Twenty of the 34 (58.8\%) patients were colonised by S. aureus that was characterised as methicillin-resistant S. aureus

108 (MRSA) in two of the patients. spa-types are given in Supporting Information Table S1. 109 Propionibacterium avidum and Propionibacterium granulosum, were present in 58.8\% and 50.0\% of 110 patients, respectively. These seven species were categorised as part of the cultivable core microbiome. 111 All other species cultivated were found in less than $50 \%$ of patients. Moreover, apart from S. epidermidis, other coagulase-negative staphylococci (CoNS) were

113 identified. Amongst those, Staphylococcus haemolyticus (44.1\% of patients), Staphylococcus capitis 114 and Staphylococcus hominis (41.2\% of patients, respectively), Staphylococcus warneri (32.4\% of 115 patients) and Staphylococcus lugdunensis (26.5\% of patients) were the most prevalent. 

23 different species distributed among 33/34 patients. Besides C. accolens and C. tuberculostearicum, most prevalent species were Corynebacterium amycolatum (20.6\% of patients), Corynebacterium aurimucosum and Corynebacterium propinquum (17.6\% of patients, respectively).

The Streptococcus genus was represented by eleven different species with the most prevalent members Streptococcus mitis/oralis (17.6\% of patients) and Streptococcus parasanguinis (11.8\% of patients) both belonging to the viridans group of streptococci (VGS). Streptococcus pneumonia appeared in 4 individuals (11.8\%), all without chronic local inflammation of the nose. Propionibacterium were found in great extend throughout all patients. Moreover, strictly anaerobes comprised members of the genera Clostridium, Anaerococcus, Finegoldia, Parvimonas, Peptoniphilus, Veillonella and Fusobacterium. Among those, Finegoldia magna was the most common isolate (38.2\% of patients).

Overall, the majority $(80.9 \%, \mathrm{n}=114)$ of all cultivable bacterial taxa were detected in only less than 5 patients. Of these, the highest fraction (48.9\%) was found in no more than one patient, 16.3\% were isolated from two, 9.2\% from three and 6.4\% from four different patients (Supporting Information Table S2).

Culturome analysis of the different nasal habitats

When analysed by culture, all four nasal habitats contained similar numbers of bacterial taxa, ranging from 8.1 (inferior meatus) to 8.7 (middle meatus) different species/genera per habitat on average

138 (Table 1). This was further confirmed when distinguishing between the patient groups. No significant variations in the number of different taxa were apparent throughout the nasal habitats if groups were

140 stratified according to gender or S. aureus carriage (Table 1, Supporting Information Table S3).

142 be found throughout all nasal habitats. Of these, only Acinetobacter lwoffii and Corynebacterium 143 simulans were not found in all four habitats (Fig. 1A). 

its carriers. In 14 of the 20 individuals (70.0\%) colonised by S. aureus, this species was present in all nasal sites; one patient showed carriage in the posterior vestibule and both nasal meatuses, one patient was colonised in the anterior and posterior vestibule and in four patients, S. aureus was exclusively isolated from the posterior vestibules (Supporting Information Table S4). Thus, the posterior vestibules were colonised in $100 \%$ of individuals who were categorised as S. aureus carriers. In fact, microbial fingerprints were generally more similar between sites within the same host, as can be seen in the nMDS plot, where there is a high similarity between the different nasal habitats by revealing visible clusters being built by all samples within each individual (Fig. 2). When samples in the same plot were marked according to the nasal site where they had been collected from, no significant pattern was observed (Supporting Information Fig. S1A).

155

Analysis of the co-existence of cultivable members of the nasal microbiota

In order to investigate the co-occurrence patterns of species, cluster analysis was used to determine how often (across samples) these groups of species co-inhabit together (Field et al., 1982). When species cluster together on the dendrogram at a high percent similarity, this indicates that these species are almost always present together in the same samples. The most prevalent species of the nasal cavities, S. epidermidis, P.acnes, C. accolens, C.tuberculostearicum, S. aureus, P. avidum and P. granulosum, formed the core community of the cultivable part of the nasal microbiota together with

163 the anaerobic species F. magna, showing a similarity level of $>50 \%$ (Fig. 3). The species 164 S. epidermidis and P. acnes appeared together with S. aureus at a similarity level of $68.4 \%$. Notable 165 simultaneous colonisation was also observed for distinct pairs of species: Dolosigranulum pigrum and Corynebacterium propinquum (66.7\%), Staphylococcus capitis and Micrococcus luteus (64.0\%) and Streptococcus mitis/oralis and Actinomyces odontolyticus (54.6\%). However, these species were found to be present together with the core members at a similarity level of less than $50 \%$ (Fig. 3). 
171 In the cultivable-fraction of the microbial community, samples from patients with chronic nasal

172 inflammation did not show clear differences to those of individuals without signs of inflammation 173 when being arranged in a nMDS plot (Supporting Information Fig. S1B). This was confirmed by an 174 ANOSIM test showing a global R of 0.375 and thus arguing for strong overlapping of bacterial communities from the two groups $(\mathrm{p}=0.029)$..Further comparison of the presence of distinct taxa

176 demonstrated that all species showing frequent presence throughout patients (detected in $\geq 5$ patients)

177 were also always detected in both patient groups (Fig. 1B). Thus, none of these frequently occurring 178 species was exclusively connected to a distinct patient group. Overall, patients with and without nasal 179 inflammatory complaints presented similar presence of members from the three main phyla of Actinobacteria, Firmicutes and Proteobacteria (Supporting Information Table S4).

181

The individual nasal cultivable microbiome

183 There was a visible similarity between the microbial communities of single individual samples at the 184 distinct nasal sites (Fig. 2). Variation in communities between different hosts appeared to be stronger than intraindividual differences in the nasal microbiome at different sites, an observation confirmed by ANOSIM (global $\mathrm{R}=0.842$, $\mathrm{p}=0.001$ ). Other factors like $S$. aureus carriage, gender, age, allergies, use of nose sprays and smoking habits did not show any clear influence on the arrangement of samples in non-metric multidimensional scaling (data not shown).

Cultivable fingerprint vs. molecular fingerprint

191 For a subset of five patients each with and without signs of inflammation, a third sampling set was 192 collected during surgery for molecular fingerprinting via Illumina 16S rRNA gene paired end sequencing (Supporting Information Table S6). The comparative analysis of the culturomic and the molecular data revealed that only a small amount of phylotypes/isolates $(n=11)$ matched in both

195 identification methods (Table 2, Fig. 4, Supporting Information Table S7). Higher numbers were 196 identified exclusively in either the culturome $(n=53)$ or the molecular data set $(n=113)$ (Table 2). 197 Analysing the resolution of both approaches, the high fidelity of species identification by cultivation 198 was demonstrated as expected. Of the 64 different taxa identified via cultivation, 62 (96.9\%) could be 
199 tracked down to their species level. While the molecular approach yielded a higher number of different 200 phylotypes $(\mathrm{n}=124)$, only $33(26.6 \%)$ were identified to species level (Table 3). However, 201 considering that only 160 bases of the 16S rRNA gene were used in this analysis, the sensitivity of the 202 method needs to be appreciated. Moreover, as shown independently for the culturome, the molecular 203 approach also yielded an individual pattern for each patient included (data not shown).

204 


\section{Discussion}

207 There is growing interest in understanding the human body surfaces as an exceptionally complex and 208 highly varying microbiological environment. Also for the nose, efforts have been made to elucidate its microbial diversity (Wos-Oxley et al., 2010; Yan et al., 2013) as well as interspecific interactions of its core members (Uehara et al., 2000; Lina et al., 2003) and other factors (Cole et al., 2001; Johannessen et al., 2012) that might play a role, especially in connection with S. aureus colonisation.

Here, we compared the nasal microbiota of two distinct groups of patients who were admitted to an ENT department: (i) people suffering from chronic nasal inflammatory diseases and (ii) people without any signs of inflammation. Using an extensive culturomic approach, we were able to isolate more than five times the number of species that can usually be detected via standard routine cultivation (Feazel et al., 2012) and more than four times the number obtained by prior cultivation studies (Alvarez et al., 2013). Composition of the most prevalent species comprising the core community in our study is in agreement with previous data reported in both, culture-based and molecular, microbiome studies (Frank et al., 2010; Wos-Oxley et al., 2010; Feazel et al., 2012; Alvarez et al., 2013; Yan et al., 2013).

Since the human nostrils are easily accessible for specimen sampling, most studies for determination of nasal S. aureus carriage were restricted to the more or less precisely defined "anterior nares” (Gillespie, 1939). Thus, the "anterior nares" have been considered as the principal habitat of S. aureus and swabbing of this region has become a main part of MRSA screening procedures. Here, a surgical approach has been chosen (i) to reach posterior areas of the nasal cavity and (ii) to avoid any contamination with the skin around the nasal ostium. Cole et al. (2001) showed that S. aureus was localised primarily to moist squamous epithelium on the septum adjacent to the nasal ostium. Also in our study, S. aureus was always detected in the vestibule, however, only $15 / 20$ (75.0\%) of the individuals tested S. aureus-positive in at least one of the four sampling sites showed colonisation of

230 the anterior region of the vestibule while all were S. aureus-positive for the posterior vestibule.

231 Consequently, the principle habitat definition for S. aureus should be extended to the complete vestibule area. Furthermore, S. aureus nasal eradication strategies should also cover posterior regions 
233 of the complete nasal cavity (vestibulum nasi and nasal meatuses) by the use of appropriate 234 formulations of the topical agent applied. Our finding that S. aureus could be found throughout all 235 nasal sites tested is supported by findings of recent investigations that also consider the different 236 anatomical parts (Yan et al., 2013). Compared to current literature, the incidence of S. aureus carriers 237 in the study group was higher than expected (Sollid et al., 2014). Data in the literature are often based 238 on simple swabbing techniques of a single nasal site (Wertheim et al., 2006; Mertz et al., 2009). Here, 239 for the first time, different parts of the human nose were precisely analyzed with a standardised 240 surgical approach applying extensive culture. The S. aureus clonal structure was very diverse (24 241 isolates with 21 spa types) and three novel spa types were detected (t14141, t14142, and t14143). Of 242 interest, one of the two MRSA isolates detected (spa type t034), belonged to the ST398 clonal lineage 243 which comprises livestock-associated MRSA known to occur on pig farms in Northwestern Germany 244 and found to enter the hospitals in this region (Köck et al., 2009, 2013). Amongst nasal specimen analysed by culture, 11 CoNS species were detected with most prevalent species being in accordance with prior findings (Becker et al., 2006; Piette and Verschraegen, 2009). Noteworthy, a relatively high presence of S. lugdunensis was observed. Despite being a member of the CoNS, similar to S. aureus, S. lugdunensis shows a high potential to cause aggressive and fast proceeding infections (Seifert et al., 2005; Piette and Verschraegen, 2009; Liu et al., 2010). Both species also share the ability to express a clumping factor and to produce a thermostable DNAse (Becker et al., 2014). Hitherto, it has been assumed, that the principal habitat of S. lugdunensis is largely restricted to the inguinal or pelvic area (Bellamy and Barkham, 2002; van der Mee-Marquet et al., 2003; Hellbacher et al., 2006). Considering the high prevalence of S. lugdunensis in our samples, the human nose may be taken into consideration as a natural habitat of equal value. Of more recently described novel staphylococcal species, only S. pettenkoferi was found in one nasal location of one individual (Trülzsch et al., 2007).

For S. pneumoniae it has been shown, that the highest rate of nasopharyngeal colonisation takes place in an early stage of life (Bogaert et al., 2004; Regev-Yochay et al., 2004). This corresponds to the low number of S. pneumonia carriers in our study, as only adult individuals were sampled. However, other members of the Streptococcus genus, mainly VGS, were isolated from more than a 
261 third of individuals in this group. As VGS contribute to the common flora to the upper respiratory tract 262 (Hardie and Whiley, 1997; Aas et al., 2005; Nakajima et al., 2013), a regular nasal presence is 263 plausible.

As expected, M. luteus, a coloniser of the human skin, was the most prevalent "micrococcal" species found in 11/34 (32.4\%) patients by culture. Newly established species of the Micrococcaceae and Dermacoccaceae families (suborder Micrococcineae), namely Kocuria koreensis, Kocuria palustris, Kocuria rhizophila and Kytococcus schroeteri were found in a much less extend below 10\%. Of interest, these "micrococcal" species have been originally associated with an occurrence in the environment or fermented food, however, human infections have been recently described for K. rhizophila and K. schroeteri (Kovács et al., 1999; Becker et al., 2008; Park et al., 2010; Moissenet et al., 2012).

The Corynebacteria genus was the most species-rich taxon in this study, comprising 23 different species and being present in almost every patient sampled. Only one individual did not show colonisation in any of the nasal habitats. Corynebacteria are being considered as part of the normal skin flora, but are highly present especially in moist regions (Grice and Segre, 2011).

Interestingly, despite the absolute number of isolates per enterobacterial species was low, more than half of the number of the individuals harboured enterobacterial species of the genera Citrobacter, Enterobacter, Escherichia, Klebsiella, Pantoea, Proteus and Serratia. Thus, the nasal cavity may represent a so far neglected reservoir for the occurrence of Enterobacteriaceae, which might be of importance for the surveillance of isolates expressing extended spectrum beta lactamases (ESBL) and carbapenemases. Nonfermenter of hospital hygienic and therapeutic concern, such as Pseudomonas aeruginosa and Stenotrophomonas maltophilia, occurred in only one and two patients, respectively.

Though the nasal cavity is well ventilated, there were considerable amounts of moderate as well as strictly anaerobic species vital throughout all nasal sites. Whilst former studies failed to detect strictly anaerobic species like F. magna (Feazel et al., 2012; Alvarez et al., 2013), known to be common members of the nasal microbiome (Wos-Oxley et al., 2010), with this approach, those species were frequently detected. 

diversity influenced by epidermal surface constitutions. Neither the variety of the different nasal habitats nor chronic inflammation had a visible impact on the microbial composition. Whereas there was no clear prove of habitat- and/or inflammation-associated differences, individual host-specific patterns of microbiota composition were revealed (Fig. 2). Most recently, such host-specific microbial structures have been shown to be already present at a very early stage of life (Sakwinska et al., 2014). Of note, other aspects such as ethnic origin, age, allergies or medical use should still be taken into account as probable drivers for these individual microbial "fingerprints" of the nasal microbiota and should be investigated in a higher resolution in following studies. While metagenomic data emerge increasingly, there is an obvious lack of comparative analysis of this method with other techniques. In this study, we were able to cultivate species that could not be found via next generation sequencing and to identify a higher number of isolates down to their species level (Table 3). These results are generally in line with former comparative studies about different techniques to analyse the human gut microbiome (Lagier et al., 2012). Indeed, it should be noted that molecular data can give a qualitative analysis of the composition of the microbiome, and typically characterise the community structure down to a certain level of abundance, whereas culturebased studies more easily identify low abundant but easy to culture members of the community. Applying state-of-the-art highly specific identification tools, an in-depth inventory of the bacterial species occurring on the surfaces of the human nasal cavity was done by culture and, in part, by 16S rRNA gene community profiling. For the first time, a standardised surgical approach was chosen for that purpose to avoid any contamination with the microbiota of the skin outside of the nasal cavity. The culturomic data revealed fingerprint-like, individual host-specific nasal microbiota that are characterised by a consistent species distribution throughout the different nasal habitats analysed. These bacterial communities appeared irrespective of local inflammation status, gender and S. aureus 313 carrier state. By cultivation, numerous species were detected that were not identified by molecular 314 analysis at the chosen resolution level. It was thus demonstrated that an extensive cultural approach in 315 combination with molecular analysis is indispensable for a detailed analysis of microbial structures of 316 a given habitat. Thorough analysis of the composition of the nasal microbiota is precondition for 
317 studies investigating the interactions of their members and their putative influence on the colonisation

318 with the major human pathogen S. aureus. The definition of the principle habitat for S. aureus should 319 be extended to the complete vestibule area particularly including the posterior regions.

320 


\section{Experimental procedures}

Study design

324 The study was conducted from October 2011 to April 2013 with full written consent of patients (file number 2010-468-f-S) admitted to the Department of Otorhinolaryngology Head and Neck Surgery

326 (ENT) of the University Hospital Münster. Nasal swab samples from two patient groups comprising

327 (i) 18 individuals with signs of chronic inflammation of nasal sites and (ii) those that were categorised 328 as non-inflamed $(n=16)$ were systematically collected during surgery. The last group included 329 patients without any signs of acute or chronic local or systemic inflammation intended for surgical corrections of innate or traumatic nasal deformations. Each dual swab sample (Transwab® Amies Duo Plain MW169P, MWE, Corsham Wiltshire, United Kingdom) was taken precisely from four defined locations in the nasal cavity: the anterior and posterior vestibules comprising skin-like squamous epithelium as well as the inferior and middle meatuses lined by ciliated mucosa. From a randomly chosen subset of ten patients, a third set of swab samples (SK-2S, Isohelix, Harrietsham, United Kingdom) was taken and used for molecular fingerprinting.

\section{Cultivation techniques}

338 After sampling, dual swabs were divided and immediately placed into either BHI medium (Becton

339 Dickinson, Franklin Lakes, NJ) supplied with 0.2\% TWEEN 80 (Merck, Darmstadt, Germany) for 340 aerobic incubation or into thioglycollate medium (Heipha Dr. Müller GmbH, Eppelheim, Germany) 341 for anaerobic cultivation. Transport tubes were sealed gas-tight and kept at room temperature 342 (max. 1 h) until they were further processed. Samples were plated onto Columbia blood agar (Becton 343 Dickinson) with 5\% sheep blood (Oxoid, Wesel, Germany), chocolate agar (Becton Dickinson) with 344 10\% sheep blood (Oxoid), Schaedler agar with 5\% sheep blood (Becton Dickinson) and Schaedler 345 agar supplied with Kanamycin, Vancomycin and 5\% horse blood (Oxoid). All agar plates were 346 incubated at $37^{\circ} \mathrm{C}$ either under aerobic, $\mathrm{CO}_{2}$ enriched (5\%) or anaerobic conditions. Swabs were also 347 suspended in either BHI medium supplied with 0.2\% TWEEN 80 for aerobic incubation overnight or 348 thioglycollate medium for anaerobic incubation for $48 \mathrm{~h}$. After incubation, $100 \mu \mathrm{l}$ aliquots of the liquid 
cultures were serially diluted up to $10^{-5}-10^{-7}$ and inoculated onto Columbia blood agar with $5 \%$ sheep blood or Schaedler agar with $5 \%$ sheep blood. These plates were incubated at $37^{\circ} \mathrm{C}$ under aerobic or anaerobic conditions.

All agar plates were read after $24 \mathrm{~h}, 48 \mathrm{~h}, 5$ days, 7 days and 14 days according to the respective cultivation condition. Different phenotypes were isolated and further grown as pure cultures (Supporting Information Fig. S2). Isolates were kept at $-80^{\circ} \mathrm{C}$ in cryo tubes (Cryobank ${ }^{\mathrm{TM}}$ System, Mast Diagnostica, Rheinfeld, Germany) for long-term storage.

\section{Identification}

358 For identification of isolates down to the species level, matrix-assisted laser desorption/ionisationtime-of-flight mass spectrometry (MALDI-TOF MS) was applied as described elsewhere (Idelevich et al., 2014). Briefly, single colonies of freshly grown pure cultures were harvested with a sterilised toothpick and smeared on a ground steel target plate (Bruker, Daltonics, Billerica, MS) in two replicates. Samples were covered with $1 \mu \mathrm{l}$ of HCCA matrix solution (alpha-cyano-4hydroxycinnamic acid solved in $50.0 \%$ acetonitrile and $2.5 \%$ trifluoroacetic acid). After cocrystallisation they were processed in the MALDI-TOF mass spectrometer (Microflex, Bruker Daltonics) using the flexControl software (flexControl 3.3, Bruker Daltonics) according to manufacturer's instructions. Spectra were analysed by MALDI Biotyper 4.0 (Bruker Daltonics) in the $\mathrm{m} / \mathrm{z}$ range of $4000-10000 \mathrm{Da}$. Isolates were considered as explicitly identified on the species level when yielding a score of $\geq 2.0$.

Members of Fusobacterium sp., Klebsiella sp., Escherichia sp. and all streptococci performing $\alpha$-hemolysis were also characterised biochemically by VITEK®2 (bioMérieux, Marcy l'Etoile, France) applying VITEK®2 GP ID cards or VITEK®2 GN ID cards (bioMérieux), respectively. Streptococci were screened for their resistance to optochin to distinguish pneumococci. Determination of

373 methicillin resistance ( $m e c A, m e c C$ ) of $S$. aureus isolates were verified by respective PCR procedures 374 as published (Becker et al., 2006; Kriegeskorte et al., 2012). spa typing was done as described 375 elsewhere (Mellmann et al., 2006). 
In the case of ambiguous MALDI-TOF MS and biochemical identification results,

377 respectively, 16S rRNA gene sequencing was performed as described before (Becker et al., 2004;

378 Breitkopf et al., 2005). Briefly, total genomic DNA was extracted using the QiaAmp MiniKit 379 according to manufacturer's instructions (Qiagen, Venlo, Netherlands). Primers $29 \mathrm{f}$ and $907 \mathrm{r}(\mathrm{m})$ were 380 used to target the V1-V4 hypervariable regions of the 16S rRNA gene (Breitkopf et al., 2005; Lane, 381 1991). PCR products were also sequenced with these primers (MWG Eurofins, Ebersberg, Germany) 382 and the obtained 16S rRNA gene sequences were aligned with SeqMan Pro ${ }^{\mathrm{TM}}$ (DNASTAR, Madison, 383 WI). Comparison of sequences was carried out in the RDP-II database (Wang et al., 2007) using the 384 Seqmatch program against sequences obtained from validly described type strains and isolates. 385 Sequences were assigned on species level at a similarity score of $\geq 98 \%$.

\section{Molecular fingerprinting via 16S rRNA gene Illumina paired-end sequencing}

388 A subset of nasal samples from ten patients was analysed by barcoded 16S rRNA gene Illumina 389 paired-end sequencing. For this, the dry swab was extracted for DNA, the bacterial 16S rRNA gene 390 amplified, amplicon libraries prepared, and bioinformatic analysis carried out as previously described 391 (Camarinha-Silva et al., 2014). In brief, primers 27F and 338R were used to target the V1-2 regions of the 16S rRNA gene, where in addition the forward primer contains a 6 nucleotide (nt) barcode and a 2 nt CA linker, and both primers comprise sequences complementary to the Illumina specific adaptors

394 to the 5'-ends. Each sample is amplified with a forward primer containing a unique barcode, so that 395 derived sequences can be sorted into their respective samples bioinformatically in downstream analysis. For evaluation of sequence quality and possible sequencing errors, positive control samples containing equimolar concentrations of established 16S rRNA gene sequences from S. aureus (DSM3463), C. accolens (GU074966), M. nonliquefaciens (JF927886) and S. epidermidis (JF927883) were used, as described in Camarinha-Silva et al., 2014. These additional samples were treated together with the other nasal samples and pooled in the final library. DNA libraries were sequenced on

401 an Illumina MiSeq platform (Illumina Inc., San Diego, CA) using $250 \mathrm{nt}$ paired-end sequencing 402 chemistries, although all sequences were trimmed to $80 \mathrm{nt}$ to account for sequence deterioration giving $403160 \mathrm{nt}$ of paired end sequence information for downstream analysis. In total, 477205 sequence reads 
were obtained. Sequences were discarded if they returned a low Phred quality score, comprised a N character in their sequence, any mismatches within the primers and barcodes or more than 10 homopolymer stretches (Camarinha-Silva et al. 2014). After filtering, samples contained a mean of 7192 sequence reads (min of 877 , max of 36355).

408

Statistical analysis

410 In order to compare the microbial fingerprints of each individual person across conditions and sites, 411 non-parametric multivariate statistical analysis was conducted with PRIMER v6 (Clarke, 1993; Clarke 412 and Gorley, 2006). For comparison of the microbial fingerprints across samples within predefined 413 groups (individual hosts or inflammation status), a sample-similarity matrix was generated using the 414 Bray-Curtis coefficient (Bray and Curtis, 1957). Samples were then ordinated using non-metric 415 multidimensional scaling (nMDS) with 50 restarts. Predefined groups of samples were tested for 416 differences via an Analysis of Similarity (ANOSIM) test using 999 permutations. Samples were 417 considered to be significantly different when the accompanying p-value was $<0.05$. The associated $\mathrm{R}$ 418 statistic ranging from 0 to 1 gave further information pertaining to the degree of separation between 419 groups of samples (Clarke and Warwick, 2001). In order to assess the co-occurrence nature of species across these samples, a species-similarity matrix was generated using the Bray-Curtis coefficient.

421 Most prevalent species (present in $\geq 5$ patients) were clustered using group-average agglomerative 422 hierarchical clustering (Field et al., 1982; Bryan, 2010; Arantes et al., 2009). When the cultivable423 fingerprinting method was analysed, the data matrix comprised presence/absence data (a binary 424 matrix). 


\section{Acknowledgements}

427

428 This work was supported by grants from the German Ministry for Education and Research (BMBF) to 429 KB and CR (0315832A) and DP (0315832B) within the joined project "The Microbiota of the Human 430 Nose Habitats - Metagenomic Analyses of their Composition and Dynamics (MENAGE)” of the 431 “Medizinische Infektionsgenomik” call.

432 The authors thank Martina Schulte, Susann Schütze, Melanie Bach and Daniela Kuhn for 433 excellent technical assistance, Ruy Jáuregui for support in 16S rRNA gene amplicon analyses and 434 Jürgen-Theodor Fränzer for surgical data supply.

435

436 Conflict of Interest

437

438 The authors declare no conflict of interest.

439 


\section{References}

441

442

443

444

445

446

447

448

449

450

451

452

453

454

455

456

457

458

459

460

461

462

463

464

465

466

467

Aas, J.A., Paster, B.J., Stokes, L.N., Olsen, I., and Dewhirst, F.E. (2005) Defining the normal bacterial flora of the oral cavity. J. Clin. Microbiol. 43: 5721-32.

Alvarez, A.S., Remy, L., Allix-Béguec, C., Ligier, C., Dupont, C., Leminor, O., et al. (2013) Patient nostril microbial flora: individual-dependency and diversity precluding prediction of Staphylococcus aureus acquisition. Clin. Microbiol. Infect. 20: 70-8.

Arantes, R.C.M., Castro, C.B., Pires, D.O., and Seoane, J.C.S. (2009) Depth and water mass zonation and species associations of cold-water octocoral and stony coral communities in the southwestern Atlantic. Mar. Ecol. Prog. Ser. 397: 71-79.

Becker, K., Harmsen, D., Mellmann, A., Meier, C., Schumann, P., Peters, G., and von Eiff, C. (2004) Development and evaluation of a quality-controlled ribosomal sequence database for $16 \mathrm{~S}$ ribosomal DNA-based identification of Staphylococcus species. J. Clin. Microbiol. 42: 49884995.

Becker, K., Heilmann, C., and Peters, G. (2014) Coagulase-Negative Staphylococci. Clin. Microbiol. Rev. 27: 870-926.

Becker, K., Pagnier, I., Schuhen, B., Wenzelburger, F., Friedrich, A.W., Kipp, F., et al. (2006) Does nasal cocolonization by methicillin-resistant coagulase-negative staphylococci and methicillinsusceptible Staphylococcus aureus strains occur frequently enough to represent a risk of falsepositive methicillin-resistant S. aureus determinations by molecular methods. J. Clin. Microbiol. 44: 229-31.

Becker, K., Rutsch, F., Uekötter, A., Kipp, F., König, J., Marquardt, T., et al. (2008) Kocuria rhizophila adds to the emerging spectrum of micrococcal species involved in human infections. J. Clin. Microbiol. 46: 3537-9.

Van Belkum, A., Verkaik, N.J., de Vogel, C.P., Boelens, H.A., Verveer, J., Nouwen, J.L., et al. (2009) Reclassification of Staphylococcus aureus nasal carriage types. J. Infect. Dis. 199: 1820-6.

Bellamy, R. and Barkham, T. (2002) Staphylococcus lugdunensis infection sites: predominance of abscesses in the pelvic girdle region. Clin. Infect. Dis. 35: E32-4. 
Bogaert, D., van Belkum, A., Sluijter, M., Luijendijk, A., de Groot, R., Rümke, H.C., et al. (2004) Colonisation by Streptococcus pneumoniae and Staphylococcus aureus in healthy children. Lancet 363: 1871-2.

Bray, J.R. and Curtis, J.T. (1957) An Ordination of the Upland Forest Communities of Southern Wisconsin. Ecol. Monogr. 27: 325.

Breitkopf, C., Hammel, D., Scheld, H.H., Peters, G., and Becker, K. (2005) Impact of a molecular approach to improve the microbiological diagnosis of infective heart valve endocarditis. Circulation 111: 1415-1421.

Bryan, M.G. (2010) Assemblages and Distributions of Groundfish Species off the West Coast of Vancouver Island. Open Mar. Biol. J. 4: 101-114.

Camarinha-Silva, A., Jáuregui, R., Chaves-Moreno, D., Oxley, A.P.A., Schaumburg, F., Becker, K., et al. (2014) Comparing the anterior nare bacterial community of two discrete human populations using Illumina amplicon sequencing. Environ. Microbiol.

Camarinha-Silva, A., Jáuregui, R., Pieper, D.H., and Wos-Oxley, M.L. (2012) The temporal dynamics of bacterial communities across human anterior nares. Environ. Microbiol. Rep. 4: 126-32.

Cheesman, K. and Burdett, E. (2011) Anatomy of the nose and pharynx. Anaesth. Intensive Care Med. 12: 283-286.

Clarke, K.R. (1993) Non-parametric multivariate analyses of changes in community structure. Aust. J. Ecol. 18: 117-143.

Clarke, K.R. and Gorley, R.N. (2006) PRIMER v6: User Manual/Tutorial. PRIMER-E, Plymouth. Prim. Plymouth.

Clarke, K.R. and Warwick, R.M. (2001) Change in Marine Communities: An Approach to Statistical Analysis and Interpretation, 2nd Edition Primer-E, Plymouth.

Cole, A.M., Tahk, S., Oren, A., Yoshioka, D., Kim, Y.H., Park, A., and Ganz, T. (2001) Determinants of Staphylococcus aureus nasal carriage. Clin. Diagn. Lab. Immunol. 8: 1064-9.

Dantes, R., Mu, Y., Belflower, R., Aragon, D., Dumyati, G., Harrison, L.H., et al. (2013) National burden of invasive methicillin-resistant Staphylococcus aureus infections, United States, 2011. JAMA Intern. Med. 173: 1970-8. 
Edgar, R.C. (2004) MUSCLE: multiple sequence alignment with high accuracy and high throughput. Nucleic Acids Res. 32: 1792-7.

Von Eiff, C., Becker, K., Machka, K., Stammer, H., and Peters, G. (2001) Nasal carriage as a source of Staphylococcus aureus bacteremia. N. Engl. J. Med. 344: 11-6.

Feazel, L.M., Robertson, C.E., Ramakrishnan, V.R., and Frank, D.N. (2012) Microbiome complexity and Staphylococcus aureus in chronic rhinosinusitis. Laryngoscope 122: 467-72.

Field, J.G., Clarke, K.R., and Warwick, R.M. (1982) A Practical Strategy for Analysing Multispecies Distribution Patterns. Mar. Ecol. Prog. Ser. 8: 37-52.

Frank, D.N., Feazel, L.M., Bessesen, M.T., Price, C.S., Janoff, E.N., and Pace, N.R. (2010) The human nasal microbiota and Staphylococcus aureus carriage. PLoS One 5: e10598.

Geurkink, N. (1983) Nasal anatomy, physiology, and function. J. Allergy Clin. Immunol. 72: 123-128.

Gillespie, E. (1939) Pathogenic staphylococci their incidence in the nose and on the skin. Lancet 234: 870-873.

Gittelman, P.D., Jacobs, J.B., Lebowitz, A.S., and Tierno, P.M. (1991) Staphylococcus aureus nasal carriage in patients with rhinosinusitis. Laryngoscope 101: 733-737.

Glück, U. (2001) Nasal carriage of Staphylococcus aureus. N. Engl. J. Med. 344: 1399; author reply $1400-1$.

Grice, E.A. and Segre, J.A. (2012) The human microbiome: our second genome. Annu. Rev. Genomics Hum. Genet. 13: 151-70.

Grice, E.A. and Segre, J.A. (2011) The skin microbiome. Nat. Rev. Microbiol. 9: 244-53.

Hardie, J.M. and Whiley, R.A. (1997) Classification and overview of the genera Streptococcus and Enterococcus. Soc. Appl. Bacteriol. Symp. Ser. 26: 1S-11S.

Hellbacher, C., Törnqvist, E., and Söderquist, B. (2006) Staphylococcus lugdunensis: clinical spectrum, antibiotic susceptibility, and phenotypic and genotypic patterns of 39 isolates. Clin. Microbiol. Infect. 12: 43-9.

Idelevich, E.A., Schüle, I., Grünastel, B., Wüllenweber, J., Peters, G., and Becker, K. (2014) Rapid identification of microorganisms from positive blood cultures by MALDI-TOF mass spectrometry subsequent to very short-term incubation on solid medium. Clin. Microbiol. Infect. 
Johannessen, M., Sollid, J.E., and Hanssen, A.-M. (2012) Host- and microbe determinants that may influence the success of S. aureus colonization. Front. Cell. Infect. Microbiol. 2: 56.

Kluytmans, J.A.J.W., van Belkum, A., and Verbrugh, H. (1997) Nasal carriage of Staphylococcus aureus: epidemiology, underlying mechanisms, and associated risks. Clin. Microbiol. Rev. 10: 505-20.

Köck, R., Becker, K., Cookson, B., van Gemert-Pijnen, J.E., Harbarth, S., Kluytmans, J., et al. (2010) Methicillin-resistant Staphylococcus aureus (MRSA): burden of disease and control challenges in Europe. Euro Surveill. 15: 19688.

Köck, R., Harlizius, J., Bressan, N., Laerberg, R., Wieler, L.H., Witte, W., et al. (2009) Prevalence and molecular characteristics of methicillin-resistant Staphylococcus aureus (MRSA) among pigs on German farms and import of livestock-related MRSA into hospitals. Eur. J. Clin. Microbiol. Infect. Dis. 28: 1375-82.

Köck, R., Schaumburg, F., Mellmann, A., Köksal, M., Jurke, A., Becker, K., and Friedrich, A.W. (2013) Livestock-associated methicillin-resistant Staphylococcus aureus (MRSA) as causes of human infection and colonization in Germany. PLoS One 8: e55040.

Kosiewicz, M.M., Zirnheld, A.L., and Alard, P. (2011) Gut microbiota, immunity, and disease: a complex relationship. Front. Microbiol. 2: 180.

Kovács, G., Burghardt, J., Pradella, S., Schumann, P., Stackebrandt, E., and Màrialigeti, K. (1999) Kocuria palustris sp. nov. and Kocuria rhizophila sp. nov., isolated from the rhizoplane of the narrow-leaved cattail (Typha angustifolia). Int. J. Syst. Bacteriol. 49 Pt 1: 167-173.

Kriegeskorte, A., Ballhausen, B., Idelevich, E.A., Köck, R., Friedrich, A.W., Karch, H., et al. (2012) Human MRSA isolates with novel genetic homolog, Germany. Emerg. Infect. Dis. 18: 1016-8.

Lagier, J.-C., Armougom, F., Million, M., Hugon, P., Pagnier, I., Robert, C., et al. (2012) Microbial culturomics: paradigm shift in the human gut microbiome study. Clin. Microbiol. Infect. 18: 1185-93.

Lane, D.J. (1991) 16S/23S rRNA sequencing. In, Stackebrandt,E. and Goodfellow,M. (eds), Nucleic acid techniques in bacterial systematics. John Wiley \& Sons, Chichester, United Kingdom, pp. $115-175$. 
Lemon, K.P., Klepac-Ceraj, V., Schiffer, H.K., Brodie, E.L., Lynch, S. V, and Kolter, R. (2010) Comparative analyses of the bacterial microbiota of the human nostril and oropharynx. MBio 1:

Lina, G., Boutite, F., Tristan, A., Bes, M., Etienne, J., and Vandenesch, F. (2003) Bacterial Competition for Human Nasal Cavity Colonization: Role of Staphylococcal agr Alleles. Appl. Environ. Microbiol. 69: 18-23.

Liu, P.-Y., Huang, Y.-F., Tang, C.-W., Chen, Y.-Y., Hsieh, K.-S., Ger, L.-P., et al. (2010) Staphylococcus lugdunensis infective endocarditis: a literature review and analysis of risk factors. J. Microbiol. Immunol. Infect. 43: 478-84.

Mediavilla, J.R., Chen, L., Mathema, B., and Kreiswirth, B.N. (2012) Global epidemiology of community-associated methicillin resistant Staphylococcus aureus (CA-MRSA). Curr. Opin. Microbiol. 15: 588-95.

Van der Mee-Marquet, N., Achard, A., Mereghetti, L., Danton, A., Minier, M., and Quentin, R. (2003) Staphylococcus lugdunensis infections: high frequency of inguinal area carriage. J. Clin. Microbiol. 41: 1404-9.

Mellmann, A., Friedrich, A.W., Rosenkötter, N., Rothgänger, J., Karch, H., Reintjes, R., and Harmsen, D. (2006) Automated DNA sequence-based early warning system for the detection of methicillin-resistant Staphylococcus aureus outbreaks. PLoS Med. 3: e33.

Mertz, D., Frei, R., Periat, N., Zimmerli, M., Battegay, M., Flückiger, U., and Widmer, A.F. (2009) Exclusive Staphylococcus aureus throat carriage: at-risk populations. Arch. Intern. Med. 169: $172-8$.

Moissenet, D., Becker, K., Mérens, A., Ferroni, A., Dubern, B., and Vu-Thien, H. (2012) Persistent bloodstream infection with Kocuria rhizophila related to a damaged central catheter. J. Clin. Microbiol. 50: 1495-8.

Nakajima, T., Nakanishi, S., Mason, C., Montgomery, J., Leggett, P., Matsuda, M., et al. (2013) Population structure and characterization of viridans group streptococci (VGS) isolated from the upper respiratory tract of patients in the community. Ulster Med. J. 82: 164-8.

Park, E.-J., Roh, S.W., Kim, M.-S., Jung, M.-J., Shin, K.-S., and Bae, J.-W. (2010) Kocuria koreensis sp. nov., isolated from fermented seafood. Int. J. Syst. Evol. Microbiol. 60: 140-3. 
Pflughoeft, K.J. and Versalovic, J. (2012) Human microbiome in health and disease. Annu. Rev. Pathol. 7: 99-122.

582

583

584

585

586

587

588

589

590

591

592

593

594

595

596

597

598

599

600

601

602

603

604

605

606

607

Piette, A. and Verschraegen, G. (2009) Role of coagulase-negative staphylococci in human disease. Vet. Microbiol. 134: 45-54.

Quast, C., Pruesse, E., Yilmaz, P., Gerken, J., Schweer, T., Yarza, P., et al. (2013) The SILVA ribosomal RNA gene database project: improved data processing and web-based tools. Nucleic Acids Res. 41: D590-6.

Regev-Yochay, G., Dagan, R., Raz, M., Carmeli, Y., Shainberg, B., Derazne, E., et al. (2004) Association between carriage of Streptococcus pneumoniae and Staphylococcus aureus in children. JAMA 292: 716-720.

Saitou, N. and Nei, M. (1987) The neighbor-joining method: a new method for reconstructing phylogenetic trees. Mol. Biol. Evol. 4: 406-425.

Sakwinska, O., Bastic Schmid, V., Berger, B., Bruttin, A., Keitel, K., Lepage, M., et al. (2014) Nasopharyngeal microbiota in healthy children and pneumonia patients. J. Clin. Microbiol. 52: $1590-4$.

Seifert, H., Oltmanns, D., Becker, K., Wisplinghoff, H., and von Eiff, C. (2005) Staphylococcus lugdunensis pacemaker-related infection. Emerg. Infect. Dis. 11: 1283-6.

Sollid, J.U.E., Furberg, A.S., Hanssen, A.M., and Johannessen, M. (2014) Staphylococcus aureus: determinants of human carriage. Infect. Genet. Evol. 21: 531-41.

Tamura, K., Stecher, G., Peterson, D., Filipski, A., and Kumar, S. (2013) MEGA6: Molecular Evolutionary Genetics Analysis Version 6.0. Mol. Biol. Evol. 30: 2725-2729.

The Human Microbiome Project Consortium (2012) Structure, function and diversity of the healthy human microbiome. Nature 486: 207-14.

Trülzsch, K., Grabein, B., Schumann, P., Mellmann, A., Antonenka, U., Heesemann, J., and Becker, K. (2007) Staphylococcus pettenkoferi sp. nov., a novel coagulase-negative staphylococcal species isolated from human clinical specimens. Int. J. Syst. Evol. Microbiol. 57: 1543-8.

Uehara, Y., Nakama, H., Agematsu, K., Uchida, M., Kawakami, Y., Abdul Fattah, A.S.M., and Maruchi, N. (2000) Bacterial interference among nasal inhabitants: eradication of 

Hosp. Infect. 44: 127-33.

610

Wagenmann, M. and Naclerio, R.M. (1992) Anatomic and physiologic considerations in sinusitis. J. Allergy Clin. Immunol. 90: 419-23.

Wang, Q., Garrity, G.M., Tiedje, J.M., and Cole, J.R. (2007) Naive Bayesian classifier for rapid assignment of rRNA sequences into the new bacterial taxonomy. Appl. Environ. Microbiol. 73: $5261-7$.

Wertheim, H.F.L., van Kleef, M., Vos, M.C., Ott, A., Verbrugh, H. a, and Fokkens, W. (2006) Nose picking and nasal carriage of Staphylococcus aureus. Infect. Control Hosp. Epidemiol. 27: 863867.

Wertheim, H.F.L., Vos, M.C., Ott, A., van Belkum, A., Voss, A., Kluytmans, J.A.J.W., et al. (2004) Risk and outcome of nosocomial Staphylococcus aureus bacteraemia in nasal carriers versus non-carriers. Lancet 364: 703-5.

Wos-Oxley, M.L., Plumeier, I., von Eiff, C., Taudien, S., Platzer, M., Vilchez-Vargas, R., et al. (2010) A poke into the diversity and associations within human anterior nare microbial communities. ISME J. 4: 839-51.

Yan, M., Pamp, S.J.J., Fukuyama, J., Hwang, P.H.H., Cho, D.-Y., Holmes, S., and Relman, D.A.A. (2013) Nasal Microenvironments and Interspecific Interactions Influence Nasal Microbiota Complexity and S. aureus Carriage. Cell Host Microbe 14: 631-640.

Yilmaz, P., Parfrey, L.W., Yarza, P., Gerken, J., Pruesse, E., Quast, C., et al. (2014) The SILVA and “All-species Living Tree Project (LTP)” taxonomic frameworks. Nucleic Acids Res. 42: D643-8. 


\section{Supporting information}

631

632 Additional Supporting Information may be found in the online version of this article at the publisher's 633 web-site:

634

635 Fig. S1. Non-metric multi-dimensional scaling plot (nMDS) demonstrating similarities of the 636 cultivable part of microbial communites between samples from (A) distinct nasal locations and (B) 637 patients with and without chronic nasal inflammation. No significant differences were demonstrated 638 for the respective groups via ANOSIM (Clarke and Warwick, 2001). Kruskal 2D Stress: 0.28.

639

640 Fig. S2. Scheme of sampling sites from the human nasal cavity chosen for cultivation and molecular 641 analysis and cultivation protocol for clinical samples collected during surgery. Exemplary design for 642 one set of swab samples obtained from one single nasal site. Swabs were first plated on agar then 643 inoculated in liquid media for overnight cultivation. On the next day liquid cultures were plated onto 644 agar directly and in dilution steps $10^{-5}-10^{-7}$. AV, anterior vestibules; PV, posterior vestibules; MM, 645 middle meatus; IM, inferior meatus. *For a subset of samples from 10 patients a third set of swab samples was collected and next generation sequencing was conducted in parallel to the culturome

647

648 Table S1. Overview of patients' metadata and nasal colonisation by S. aureus as detected by culture 649 Table S2. List of all species/genera isolated by culture in the group of 34 individuals and their 651 presence in every single individual analysed

652

653 Table S3. Absolute numbers of different cultivable species/genera isolated from the different nasal 654 habitats and patients included in this study

655

656 Table S4. Distribution of S. aureus throughout patient groups and nasal habitats as found by culture 
657 Table S5. List of all species/genera isolated by culture in the group of 34 individuals and their 658 appearance in the different patient groups and nasal habitats

659

660 Table S6. List of all phylotypes detected by 16S rRNA gene community profiling in the subset of 661 samples from 10 individuals and their presence throughout all nasal samples analysed

662

663 Table S7. Comparison of species reliably identified in the subset of samples from 10 individuals via 664 cultivation and 16S rRNA gene community profiling

665 


\section{Figure legends}

667

668 Fig. 1. Prevalence of the most common cultivable nasal species (isolated from $\geq 5$ patients) in (A) the 669 different nasal habitats and (B) individuals with and without chronic inflammatory nasal diseases.

670 A: bars represent the proportion of patients carrying the respective species when being grouped into 671 individuals suffering from chronic nasal inflammation $(n=18)$ and those without inflammatory 672 complaints $(n=16)$. B: bars represent the fraction of all participants $(n=34)$ carrying the respective 673 species in either of the nasal habitats analysed. Bacillus cereus group includes isolates identified as 674 Bacillus cereus, Bacillus mycoides, Bacillus thuringiensis and Bacillus weihenstephanensis via 675 MALDI-TOF MS.

676

677 Fig. 2. Non-metric multi-dimensional scaling plot (nMDS) visualising the individual specific 678 similarities of the different nasal habitats’ cultivable microbiota.

679 Shown is an overview of all samples from the 34 patients (big box) and the individual-related samples 680 singled out from the overview (small boxes). Samples from one individual (different habitats) are 681 marked in the same colour. Triangles indicate patients suffering from chronic inflammatory nasal diseases, circles represent individuals without inflammation. Kruskal 2D Stress: 0.28. Analysis of similarities (ANOSIM) on single individual's nasal microbiota showed significant differences of the

684 interindividual nasal communities (Global $\mathrm{R}=0.842 ; \mathrm{p}=0.001$ ). AV, anterior vestibule; $\mathrm{PV}$, posterior 685 vestibule; MM, middle meatus; IM, inferior meatus.

686

687 Fig. 3. Cluster diagram of the most prevalent species/genera (detected in $\geq 5$ patients) illustrating their relative co-occurrence on patient level.

689 Position of nodes represents the relative frequency of the different species/genera appearing 690 simultaneously in the same patient. From presence/absence data on patient level of the different 691 species, a similarity matrix was generated using the Bray-Curtis coefficient. The dendrogram was 692 calculated via group-average agglomerative hierarchical clustering. Bacillus cereus group includes 
693 isolates identified as Bacillus cereus, Bacillus mycoides, Bacillus thuringiensis and Bacillus 694 weihenstephanensis via MALDI-TOF MS.

695

696 Fig. 4. Prevalence of isolates identified via culturomic analysis and 16S rRNA gene community 697 profiling onto species level in the comparative subset of samples from 10 patients. Bars represent the 698 relative number of nasal samples tested positive for the respective species via each of the methods. 699 The complete list of identifications down to species level in the subset analysed via culture dependent 700 and independent methods is given in Table S7.

701 
Table 1. Absolute numbers of cultivated species/genera observed on average per patient and nasal habitat and stratified according to inflammatory state of nasal sites, gender and S. aureus carriage state.

\begin{tabular}{|c|c|c|c|c|c|c|c|}
\hline \multirow{2}{*}{ Nasal habitat $^{\mathrm{a}}$} & \multicolumn{2}{|c|}{ Inflammatory state $^{b}$} & \multicolumn{2}{|c|}{ Gender } & \multicolumn{2}{|c|}{ S. aureus carriage ${ }^{c}$} & \multirow{2}{*}{ Total patients } \\
\hline & CI & NI & Male & Female & positive & negative & \\
\hline AV & $7.8 \pm 2.2$ & $8.5 \pm 4.1$ & $8.6 \pm 3.7$ & $7.3 \pm 1.8$ & $7.8 \pm 3.2$ & $8.6 \pm 3.2$ & $8.1 \pm 3.2$ \\
\hline PV & $8.6 \pm 3.0$ & $7.9 \pm 3.0$ & $8.2 \pm 2.8$ & $8.5 \pm 3.4$ & $8.4 \pm 3.3$ & $8.2 \pm 2.4$ & $8.3 \pm 3.0$ \\
\hline MM & $8.3 \pm 3.3$ & $9.3 \pm 4.6$ & $9.4 \pm 4.0$ & $7.5 \pm 3.7$ & $8.5 \pm 3.7$ & $9.1 \pm 4.3$ & $8.7 \pm 3.9$ \\
\hline IM & $9.2 \pm 3.4$ & $6.9 \pm 3.1$ & $8.5 \pm 2.7$ & $7.4 \pm 4.6$ & $8.4 \pm 3.8$ & $7.7 \pm 3.0$ & $8.1 \pm 3.4$ \\
\hline All habitats & $15.7 \pm 6.0$ & $14.5 \pm 6.7$ & $15.2 \pm 5.5$ & $15.0 \pm 7.6$ & $14.8 \pm 6.5$ & $15.6 \pm 6.1$ & $15.2 \pm 6.2$ \\
\hline
\end{tabular}

a. Nasal sites chosen for sampling in his study. AV, anterior vestibule; PV, posterior vestibule; MM, middle meatus; IM, inferior meatus. chronic nasal inflammation.

c. Nasal S. aureus carriage as detected by culture. 
Table 2. Comparison of cultivation and molecular analysis regarding their potential to exclusively identify distinct phylotypes/species ${ }^{\mathrm{a}}$.

\begin{tabular}{lcccccccccccc}
\hline Patient number & 7 & 10 & 15 & 26 & 29 & 4 & 14 & 19 & 22 & 30 & Total $^{\mathrm{b}}$ & $\%^{\mathrm{c}}$ \\
\hline Isolates/phylotypes exclusively identified via: & & & & & & & & & & & & \\
Culturome & 9 & 10 & 9 & 10 & 14 & 15 & 12 & 9 & 6 & 16 & 53 & 29.9 \\
Molecular fingerprinting & 30 & 52 & 40 & 25 & 25 & 48 & 21 & 15 & 22 & 32 & 113 & 63.8 \\
Both methods & 6 & 4 & 4 & 6 & 1 & 4 & 2 & 5 & 3 & 2 & 11 & 6.2 \\
Total (isolates/phylotypes) & 45 & 66 & 53 & 41 & 40 & 67 & 35 & 29 & 31 & 50 & 177 & 100.0
\end{tabular}

Species exclusively identified via:

$\begin{array}{lcccccccccccc}\text { Culturome } & 8 & 10 & 8 & 9 & 13 & 15 & 11 & 8 & 6 & 16 & 51 & 60.7 \\ \text { Molecular fingerprinting } & 8 & 15 & 14 & 3 & 8 & 14 & 7 & 3 & 8 & 9 & 22 & 26.2 \\ \text { Both methods } & 6 & 4 & 4 & 6 & 1 & 4 & 2 & 5 & 3 & 2 & 11 & 13.1 \\ \text { Total (species) } & 22 & 29 & 26 & 18 & 22 & 33 & 20 & 16 & 17 & 27 & 84 & 100.0\end{array}$

712 a. Given are the total numbers of isolates/phylotypes and species identified via either cultivation or molecular analysis and those identified via both methods 713 in each of the 10 individuals where molecular analyses have been performed.

714 b. Total amount of different isolates/phylotypes or species found in the complete subset of patients.

715 c. Relative amount of different isolates/phylotypes or species found in the complete subset of patients. 
Table 3. Comparison of quantity and resolution of species identification via cultivation and molecular analysis ${ }^{\mathrm{a}}$.

\begin{tabular}{|c|c|c|c|c|c|c|c|c|c|c|c|c|}
\hline Patient number & 7 & 10 & 15 & 26 & 29 & 4 & 14 & 19 & 22 & 30 & Total $^{\mathrm{b}}$ & $\%^{\mathrm{c}}$ \\
\hline \multicolumn{13}{|c|}{ Different isolates in culturome identified on: } \\
\hline Species level & 14 & 14 & 12 & 16 & 15 & 19 & 13 & 13 & 9 & 18 & 62 & 96.9 \\
\hline Genus level & 1 & 0 & 1 & 0 & 0 & 0 & 1 & 1 & 0 & 0 & 2 & 3.1 \\
\hline Family level & 0 & 0 & 0 & 0 & 0 & 0 & 0 & 0 & 0 & 0 & 0 & 0.0 \\
\hline No distinct level & 0 & 0 & 0 & 0 & 0 & 0 & 0 & 0 & 0 & 0 & 0 & 0.0 \\
\hline Total (isolates) & 15 & 14 & 13 & 16 & 15 & 19 & 14 & 14 & 9 & 18 & 64 & 100.0 \\
\hline \multicolumn{13}{|c|}{ Different phylotypes in molecular data set identified on: } \\
\hline Species level & 14 & 19 & 18 & 9 & 9 & 17 & 9 & 8 & 11 & 11 & 33 & 26.6 \\
\hline Genus level & 19 & 31 & 22 & 12 & 12 & 30 & 8 & 9 & 12 & 13 & 68 & 54.8 \\
\hline Family level & 2 & 4 & 3 & 3 & 4 & 2 & 4 & 2 & 2 & 7 & 11 & 8.9 \\
\hline No distinct level & 1 & 2 & 1 & 7 & 1 & 3 & 2 & 1 & 0 & 3 & 12 & 9.7 \\
\hline Total (phylotypes) & 36 & 56 & 44 & 31 & 26 & 52 & 23 & 20 & 25 & 34 & 124 & 100.0 \\
\hline
\end{tabular}

718 a. Listed are the absolute numbers of isolates/phylotypes that were identified in each of the 10 individuals where molecular analyses have been performed, as 719 well as the level of differentiation.

720 b. Total amount of different isolates/phylotypes found in the complete subset of patients.

721 c. Relative amount of different isolates/phylotypes found in the complete subset of patients. 